\title{
CENTRAL UNITS OF INTEGRAL GROUP RINGS OF NILPOTENT GROUPS
}

\author{
E. JESPERS, M. M. PARMENTER, AND S. K. SEHGAL
}

(Communicated by Ronald Solomon)

\begin{abstract}
In this paper a finite set of generators is given for a subgroup of finite index in the group of central units of the integral group ring of a finitely generated nilpotent group.
\end{abstract}

In this paper we construct explicitly a finite set of generators for a subgroup of finite index in the centre $Z(\mathbf{U}(\mathbf{Z} G))$ of the unit group $\mathcal{U}(\mathbf{Z} G)$ of the integral group ring $\mathbf{Z} G$ of a finitely generated nilpotent group $G$. Ritter and Sehgal [4] did the same for finite groups $G$, giving generators which are a little more complicated. They also gave in [2] necessary and sufficient conditions for $Z(\mathcal{U}(\mathbf{Z} G))$ to be trivial; recall that the units $\pm G$ are called the trivial units. We first give a finite set of generators for a subgroup of finite index in $Z(\mathcal{U}(\mathbf{Z} G))$ when $G$ is a finite nilpotent group. Next we consider an arbitrary finitely generated nilpotent group and prove that a central unit of $\mathbf{Z} G$ is a product of a trivial unit and a unit of $\mathbf{Z} T$, where $T$ is the torsion subgroup of $G$. As an application we obtain that the central units of $\mathbf{Z} G$ form a finitely generated group and we are able to give an explicit set of generators for a subgroup of finite index.

\section{Finite NilPotent GROUPS}

Throughout this section $G$ is a finite group. When $G$ is Abelian, it was shown in [1] that the Bass cyclic units generate a subgroup of finite index in the unit group. Using a stronger version of this result, also proved by Bass in [1], we will construct a finite set of generators from the Bass cyclic units when $G$ is finite nilpotent.

Our notation will follow that in [6]. The following lemma is proved in [1].

Lemma 1. The images of the Bass cyclic units of $\mathbf{Z} G$ under the natural homomorphism $j: \mathcal{U}(\mathbf{Z} G) \rightarrow K_{1}(\mathbf{Z} G)$ generate a subgroup of finite index.

Let $L$ denote the kernel of this map $j$, and $B$ the subgroup of $\mathcal{U}(\mathbf{Z} G)$ generated by the Bass cyclic units. It follows that there exists an integer $m$ such that $z^{m} \in L B$ for all $z \in Z(\mathcal{U}(\mathbf{Z} G))$, and so we can write $z^{m}=l b_{1} b_{2} \cdots b_{k}$ for some $l \in L$ and Bass cyclic units $b_{i}$

Received by the editors August 4, 1994

1991 Mathematics Subject Classification. Primary 16U60, 20C05, 20C07; Secondary 20C10, $20 \mathrm{C} 12$.

This work is supported in part by NSERC Grants OGP0036631, A8775 and A5300, Canada, and by DGICYT, Spain. 
Next, let $Z_{i}$ denote the $i$-th centre of $G$, and suppose from now on that $G$ is nilpotent of class $n$. For any $x \in G$ and Bass cyclic unit $b \in \mathbf{Z}\langle x\rangle$, we define

$$
b_{(1)}=b
$$

and for $2 \leq i \leq n$

$$
b_{(i)}=\prod_{g \in Z_{i}} b_{(i-1)}^{g},
$$

where $\alpha^{g}=g^{-1} \alpha g$ for $\alpha \in \mathbf{Z} G$. Note that by induction $b_{(i)}$ is central in $\mathbf{Z}\left\langle Z_{i}, x\right\rangle$ and independent of the order of the conjugates in the product expression. In particular, $b_{(n)} \in Z(\mathcal{U}(\mathbf{Z} G))$.

Recall again that if $z \in Z(\mathcal{U}(\mathbf{Z} G))$, then $z^{m}=l b_{1} b_{2} \cdots b_{k}$ for some $l \in L$ and Bass cyclic units $b_{i}$. Since $K_{1}(\mathbf{Z} G)$ is Abelian, we can write

$$
\begin{array}{rlrl}
z^{m\left|Z_{2}\right|\left|Z_{3}\right| \cdots\left|Z_{n}\right|} & =\left(l b_{1} b_{2} \cdots b_{k}\right)^{\left|Z_{2}\right|\left|Z_{3}\right| \cdots\left|Z_{n}\right|} & \\
& =l_{1} \prod_{1 \leq i \leq k} b_{i}^{\left|Z_{2}\right|\left|Z_{3}\right| \cdots\left|Z_{n}\right|} & & \text { for some } l_{1} \in L \\
& =l_{2} \prod_{1 \leq i \leq k} b_{i(2)}^{\left|Z_{3}\right| \cdots\left|Z_{n}\right|} & & \text { for some } l_{2} \in L \\
& =l^{\prime} \prod_{1 \leq i \leq k} b_{i(n)} & & \text { for some } l^{\prime} \in L .
\end{array}
$$

Since each $b_{i(n)}$ is in $Z(\mathcal{U}(\mathbf{Z} G))$, we conclude that $l^{\prime} \in L \cap Z(\mathcal{U}(\mathbf{Z} G))$. But we shall show next that $L \cap Z(\mathcal{U}(\mathbf{Z} G))$ is trivial, so $l^{\prime} \in \pm Z(G)$. The argument uses the same idea as in [3, Lemma 3.2].

For every primitive central idempotent $e$ in the rational group algebra $\mathbf{Q} G$, the simple ring $\mathbf{Q} G e$ has a reduced norm which we denote by $n r_{e}$. Further, denote

$$
m_{e}=\sqrt{[\mathbf{Q} G e: Z(\mathbf{Q} G e)]}
$$

and let

$$
r=\prod_{e} m_{e}
$$

Now let $l^{\prime} \in L \cap Z(\mathcal{U}(\mathbf{Z} G))$. By definition of $K_{1}(\mathbf{Z} G)$ this means that a suitable matrix

$$
\left[\begin{array}{cccccc}
l^{\prime} & & & & & \\
& 1 & & & & \\
& & \cdot & & & \\
& & & \cdots & & \\
& & & & \cdot & \\
& & & & & 1
\end{array}\right]
$$

is a product of commutators. Therefore $l^{\prime} e$ has reduced norm one. Since $l^{\prime} e$ is also central, we obtain

$$
\left(l^{\prime} e\right)^{m_{e}}=n r\left(l^{\prime} e\right) e=e
$$

Hence

$$
l^{\prime r}=1 .
$$

So $l^{\prime}$ is a torsion central unit, and therefore is trivial [7, Corollary 1.7, page 4].

Since $Z(\mathcal{U}(\mathbf{Z} G))$ is finitely generated (see, e.g., $[2]),(Z(\mathcal{U}(\mathbf{Z} G)))^{m\left|Z_{2}\right|\left|Z_{3}\right| \cdots\left|Z_{n}\right|}$ is of finite index. But we have just seen that the latter subgroup is contained in the subgroup generated by $\pm Z(G)$ and $\left\{b_{(n)} \mid b\right.$ a Bass cyclic unit $\}$. We have proved 
Proposition 2. Let $G$ be a finite nilpotent group of class $n$. Then

$$
\left.\left\langle b_{(n)}\right| b \text { a Bass cyclic }\right\rangle
$$

is of finite index in $Z(\mathcal{U}(\mathbf{Z} G))$.

Remark. Note that our method for constructing generators for a subgroup of finite index in $Z(\mathcal{U}(\mathbf{Z} G))$ can be adapted for some other classes of finite groups $G$. For example if $G=D_{2 n}=\left\langle a, b \mid x^{n}=1, y^{2}=1, y x=x^{n-1} y\right\rangle$, the dihedral group of order $2 n$, then the only nontrivial Bass cyclic units $b$ of $\mathbf{Z} D_{2 n}$ belong to $\mathbf{Z}\langle x\rangle$. It follows that $b b^{y}=b^{y} b$ is central. Our proof now remains valid and yields that $\left\langle b b^{y}\right| b$ a Bass cyclic in $\left.\mathbf{Z}\langle x\rangle\right\rangle$ is of finite index in $Z\left(\mathcal{U}\left(\mathbf{Z} D_{2 n}\right)\right)$.

\section{Finitely GENERATED NILPOTENT GROUPS}

We will now consider central units of an integral group ring of an arbitrary finitely generated nilpotent group $G$. The torsion subgroup of $G$ is denoted $T$. First we show that central units of $\mathbf{Z} G$ have the following decomposition.

Proposition 3. Let $G$ be a finitely generated nilpotent group. Every $u \in Z(\mathcal{U}(\mathbf{Z} G))$ can be written as $u=r g, r \in \mathbf{Z} T, g \in G$.

Proof. Let $F=G / T$. Since $T$ is finite and $F$ acts on the set of primitive central idempotents of $\mathbf{Q} T$ by conjugation, by adding the idempotents in an orbit of this action we obtain

$$
\mathbf{Q} T=\bigoplus(\mathbf{Q} T) e_{i}=\bigoplus R_{i},
$$

where $e_{i}$ are primitive central idempotents of $\mathbf{Q} G$. Then $\mathbf{Q} G$ is the crossed product

$$
\mathbf{Q} G=\mathbf{Q} T * F=\left(\bigoplus R_{i}\right) * F=\bigoplus R_{i} * F .
$$

Decompose $u$ as a sum of elements in $(*)$ :

$$
u=\bigoplus_{i}\left(\sum_{j=1}^{n} u_{j} f_{j}\right), \quad 0 \neq u_{j} \in R_{i}, f_{j} \in G, \text { for each } j .
$$

We assume that we have put together the $u_{j}$ 's with the same $f_{j} T \in G / T$, namely for $k \neq j, f_{k} T \neq f_{j} T$.

We claim that $n=1$. Let us denote by - the projection of $\mathbf{Q} G$ onto $R_{i} * F$. Then since $u$ is central we have $\overline{\mathbf{Q} T} \bar{u}=\bar{u} \overline{\mathbf{Q} T}$, which implies $\overline{\mathbf{Q} T} u_{j} f_{j}=u_{j} f_{j} \overline{\mathbf{Q} T}$ for all $j$. It follows that $u_{j}$ is not a zero divisor provided $R_{i}$ has only one simple (artinian) component, and so $u_{j}$ is a unit. The only time $u_{j}$ can be a nonunit is when it has some zero components in the simple components of $R_{i}$. However, by the construction of $R_{i}$, these latter components can be moved to any other place by conjugating suitably. But they must stay put due to the facts that $F$ is ordered and $\bar{u}$ is central. It follows that $u_{j}$ is a unit for all $j$. Hence, working in $R_{i} * F$ and using again that $F$ is ordered, it follows by a classical argument that $\bar{u}=\sum_{j} u_{j} f_{j}$ is simply equal to $u_{n} f_{n}$ as claimed.

Changing notation, we write

$$
u=\bigoplus_{i} \alpha f, \quad \alpha \in R_{i}, f \in G
$$


Let $k=|A u t(T)|$, so $f^{k}$ commutes with $T$ for $f \in G$. Hence

$$
u^{k}=\bigoplus(\alpha f)^{k}=\bigoplus \beta f^{k}, \quad \beta \in R_{i}
$$

(note that the number of summands in $u^{k}$ is the same as the number of summands in $u$, because each $\alpha$ is a unit in $R_{i}$ ), and thus

$$
u^{k}=\left(u^{k}\right)^{f_{1}^{k}}=\bigoplus\left(\beta f^{k}\right)^{f_{1}^{k}}=\bigoplus \beta t f^{k}, \quad t \in T .
$$

The last step follows from the fact that conjugation will preserve the order on the $f T$ 's in the ordered group $F$. Since $\left(f^{k}\right)^{f_{1}^{k}}=t f^{k}$, we can choose $k$ large enough so that all the $f^{k}$ commute with each other and with $T$. Thus we may assume that

$$
u^{k}=\bigoplus \beta f^{k} \text {. }
$$

Again, we put together all $\beta$ with the same $f^{k} T$. In other words, we assume that $u^{k}=\bigoplus \beta f^{k}$ with all $f^{k} T$ different. Note that these new values of $\beta$ all lie in $\mathbf{Z T}$. Furthermore, we now obtain for each $t \in T$,

$$
u^{k}=\left(u^{k}\right)^{t}=\bigoplus(\beta)^{t} f^{k},
$$

and thus $\beta^{t}=\beta$. So the ring $R$ generated by all the $\beta$ is commutative. Again, if necessary, replacing $k$ by a high enough power, we may assume that the group $A$ generated by all the $f^{k}$ in the summation of $u^{k}$ is a torsion-free Abelian group, and thus a free Abelian group. Consequently

$$
u^{k} \in R A,
$$

the commutative group ring of $A$ over $R$. Let $N=\operatorname{Rad}(R)$ be the set of nilpotent elements of $R$. Now $\mathbf{Z} T$ has only trivial idempotents [6, Theorem 2.20, page 25]. Hence since $R \subseteq \mathbf{Z T}$ and since idempotents of $R / N$ can be lifted to $R$, it follows that $R / N$ also has only trivial idempotents. Therefore [6, Lemma 3.3, page 55] together with an inductive argument tells us that $(R / N) A$ has only trivial units. It follows that

$$
u^{k}=\beta f^{k}+\text { nilpotent elements. }
$$

But as each $\beta$ is a sum of units in various $R_{i}$, it follows that the last term must be zero. Hence $u^{k}=\beta f^{k}$, and thus all $f$ 's in the original decomposition of $u=\bigoplus_{i} \alpha f$ were in the same coset of $T$. Thus $u=r f$ as required.

We give two important consequences of the last result. We say that $Z(\mathcal{U}(\mathbf{Z} G))$ is trivial if it contains only trivial units.

Corollary 4. Let $G$ be a finitely generated nilpotent group. If $Z(\mathcal{U}(\mathbf{Z T}))$ is trivial, then $Z(\mathcal{U}(\mathbf{Z} G))$ is trivial.

Proof. Let $u \in Z(\mathcal{U}(\mathbf{Z} G))$ be nontrivial. Then the support of $u$ contains two different elements, say $x$ and $y$. Since finitely generated nilpotent groups are residually finite, there exists a finite factor $G / N=\bar{G}$ so that $\bar{x} \neq \bar{y}$ in $\bar{G}$ (see [5, page 149]). Hence $\bar{u}$ has in its support at least two different elements, and thus $\bar{u}$ is of infinite order ([7, Corollary 1.7, page 4]). By Proposition 3 we write $u=r g, r \in \mathbf{Z T}$, $g \in G$. Since $u$ is central, $r$ commutes with $g$. It then follows easily that $\bar{r}$, and hence also $r$, is of infinite order. Moreover, there exists a positive integer $n$ such that $\left(g^{n}, T\right)=1$. Consequently it follows from $u^{n}=r^{n} g^{n}$ that $r^{n}$ commutes with $T$. Thus $r^{n}$ is a nontrivial unit of $Z(\mathcal{U}(\mathbf{Z} T))$. 
Corollary 5. Let $G$ be a finitely generated nilpotent group. Then $Z(\mathcal{U}(\mathbf{Z} G))$ is finitely generated. Furthermore, $(Z(\mathcal{U}(\mathbf{Z} G)) \cap Z(\mathcal{U}(\mathbf{Z} T))) Z(G)$ is of finite index in $Z(\mathcal{U}(\mathbf{Z} G))$.

Proof. Let $S=Z(\mathcal{U}(\mathbf{Z} G)) \cap Z(\mathcal{U}(\mathbf{Z} T))$. First we show that $Z(\mathcal{U}(\mathbf{Z} G)) / S Z(G)$ is a torsion group of bounded exponent. Indeed, let $u \in Z(\mathcal{U}(\mathbf{Z} G))$. Because of Proposition 3 write $u=r g$, with $r \in \mathcal{U}(\mathbf{Z} T)$ and $g \in G$. Considering the natural epimorphism $\mathbf{Z} G \rightarrow \mathbf{Z}(G / T)$ and using the fact that $Z(\mathcal{U}(\mathbf{Z}(G / T)))$ is trivial because $G / T$ is ordered, it follows that $g T \in Z(G / T)$. Hence $\left(g^{k}, T\right)=1$ and $g^{l} \in Z(G)$ for $k=|A u t(T)|$ and $l=k|T|$. Now since $u$ is central, $r$ and $g$ commute. Therefore

$$
u^{l}=r^{l} g^{l} \text { and } r^{l} \in S .
$$

Consequently $u^{l} \in S Z(G)$, and the claim follows.

As a subgroup of the finitely generated group $Z(\mathcal{U}(\mathbf{Z} T))$, the group $S$ is itself finitely generated. Hence so is $S Z(G)$. Since the torsion subgroup of $Z(\mathcal{U}(\mathbf{Z} G))$ is finite (see for example [6, page 46]), the above claim now easily yields that $Z(\mathcal{U}(\mathbf{Z} G))$ is indeed finitely generated.

We will now construct finitely many generators for the central units of any finitely generated nilpotent group.

Let $n$ be the nilpotency class of $T$ and $h$ the Hirsch number of $G / T$. Let $k=$ $|A u t(T)|$. Further let $x_{1}, \cdots, x_{h}$ be elements of $G$ such that for each $1 \leq i \leq h$ the group $G_{i}=\left\langle T, x_{1}, \cdots, x_{i}\right\rangle$ is normal in $G$ and $G_{i} / G_{i-1} \cong \mathbf{Z}$, where $G_{0}=T$. For any generator $b_{(n)}$ described in Proposition 2 define

and for $1 \leq i \leq h$

$$
b_{(n)}^{(0)}=b_{(n)}
$$

$$
b_{(n)}^{(i)}=\prod_{0 \leq j<k}\left(b_{(n)}^{(i-1)}\right)^{x_{i}^{j}} .
$$

Since each $b_{(n)}$ is in $Z(\mathcal{U}(\mathbf{Z} T))$, the order of the conjugates in the product expression for $b_{(n)}^{(i)}$ is unimportant. It follows by induction that $b_{(n)}^{(i)}$ is in $Z\left(\mathcal{U}\left(\mathbf{Z} G_{i}\right)\right)$. In particular, $b_{(n)}^{(h)} \in Z(\mathcal{U}(\mathbf{Z} G))$.

Theorem 6. Let $G$ be a finitely generated nilpotent group. Suppose $n$ is the nilpotency class of $T$ and $h$ is the Hirsch number of $G / T$. Then

$$
\left.\left\langle b_{(n)}^{(h)}\right| b \text { a Bass cyclic of } \mathbf{Z} T\right\rangle Z(G)
$$

is of finite index in $Z(\mathcal{U}(\mathbf{Z} G))$.

Proof. Because of Corollary 5 the group $S Z(G)$ with $S=Z(\mathcal{U}(\mathbf{Z} G)) \cap Z(\mathcal{U}(\mathbf{Z} T))$ is of finite index in $Z(\mathcal{U}(\mathbf{Z} G))$. Let $\alpha_{1}, \cdots, \alpha_{p}$ be a finite set of generators for $S$. By Proposition 2 there exists a positive integer $m$ such that all $\alpha_{1}^{m}, \cdots, \alpha_{p}^{m}$ are in $\left\langle b_{(n)}\right|$ b a Bass cyclic in $\left.\mathbf{Z} T\right\rangle$. For simplicity, write $\alpha=\alpha_{1}^{m}$. Then

$$
\alpha=\prod b_{(n)},
$$

where the product runs over a finite number of Bass cyclic units of $\mathbf{Z T}$. Since $\alpha$ is in $Z(\mathcal{U}(\mathbf{Z} G))$, and using the notation introduced above, we obtain

$$
\alpha^{k}=\alpha \alpha^{x_{1}} \cdots \alpha^{x_{1}^{k-1}} .
$$


As each $b_{(n)}$ is central in $\mathbf{Z T}$, this implies

$$
\alpha^{k}=\prod b_{(n)}^{(1)} .
$$

Continuing this process one obtains that

$$
\alpha^{k^{h}}=\prod b_{(n)}^{(h)} .
$$

Since the group generated by $\alpha_{1}^{m k^{h}}, \cdots, \alpha_{p}^{m k^{h}}$ is of finite index in $S$, the result follows.

Note that Corollary 4 can now also be obtained as an easy consequence of Theorem 6 .

We now give an example showing that the converse of Corollary 4 does not hold.

Example. Let $G=\left\langle a, x \mid a^{x}=a^{3}, a^{8}=1\right\rangle$. Clearly $G$ is a nilpotent group with $T=\langle a\rangle$, a cyclic group of order 8. From Higman's result (see [6]) it follows that $Z(\mathcal{U}(\mathbf{Z} T))$, modulo the trivial units, is a free Abelian group of rank 1 . We now show that $Z(\mathcal{U}(\mathbf{Z} G))$ contains only trivial units. For this suppose $u$ is a nontrivial central unit in $\mathbf{Z} G$. By Proposition 3, we can write $u=r x^{i}$ for some integer $i$ and $r \in \mathcal{U}(\mathbf{Z} T))$. We know from the above that $r$ is of infinite order, and since $r$ commutes with $x$, it must be in $Z(\mathcal{U}(\mathbf{Z} G))$.

Because the only Bass cyclic unit, up to inverses, in $\mathbf{Z T}$ is

$$
b=\left(1+a+a^{2}\right)^{4}-10 \hat{a}, \quad \hat{a}=1+a+\cdots+a^{7},
$$

Proposition 2 yields that

$$
r^{k}=b^{l},
$$

for some nonzero integers $k, l$. Observe, however, that $b^{x}=b^{-1}$. Since $b^{l}=r^{k}$ is central in $\mathbf{Z} G$, we obtain $b^{l}=b^{-l}$, contradicting the fact that $b$ is of infinite order.

\section{REFERENCES}

1. H. Bass, The Dirichlet Unit Theorem, Induced Characters and Whitehead Groups of Finite Groups, Topology, 4 (1966), 391-410. MR 33:1341

2. J. Ritter and S.K. Sehgal, Integral group rings with trivial central units, Proc. Amer. Math. Soc., 108 (1990), 327-329. MR 90d:16009

3. J. Ritter and S.K. Sehgal, Construction of Units in Integral Group Rings of Finite Nilpotent Groups, Trans. Amer. Math. Soc., 324 (2)(1991), 603-621. MR 91h:20008

4. J. Ritter and S.K. Sehgal, Units of group rings of solvable and Frobenius groups over large rings of cyclotomic integers, Journal of Algebra, 158 (1993), 116-129. MR 95d:16045

5. Derek J.S. Robinson, A course in the theory of groups, Springer Verlag, 1982. MR 84k:20001

6. S. K. Sehgal, Topics in group rings, Marcel Dekker, New York, 1978. MR 80j:16001

7. S. K. Sehgal, Units in integral group rings, Longman Scientific and Technical, Essex, 1993. MR 94m:16039

(E. Jespers and M. M. Parmenter) Department of Mathematics and Statistics, Memorial University of Newfoundland, St. John's, Newfoundland, Canada A1C 5S7

E-mail address: ejespers@albert.math.mun.ca

E-mail address: mparmen@plato.ucs.mun.ca

(S. K. Sehgal) Department of Mathematics, University of Alberta, Edmonton, AlBerta, CANADA T6G 2G1

E-mail address: ssehgal@schur.math.ualberta.ca 